one patient with $\mathrm{CCHB}$ one year after pace maker implanting.

Conclusions CCHB is the most serious manifestation of the NLE associated with significant morbidity and mortality. Early diagnosis can lead to early treatment avoiding in some cases the onset of CCHB.

\section{P441 YIELD OF URINE FOR CMV SCREENING TEST FOR ASYMPTOMATIC MICRO-CEPHALIC INFANTS1}

Haroon Usman*, Prof Martin White. Neonatology Department, Coombe Women and Infants University Hospital, Dublin, Ireland

\subsection{6/archdischild-2019-epa.777}

Background Congenital CMV infection is the leading cause of nonhereditary SNHL and other long-term neurodevelopmental disabilities. Its prevalence is $0.6 \%$ in developed countries. Approximately $10 \%$ infants have symptoms at birth. Among apparently asymptomatic infants, approximately 10 to $15 \%$ experience SNHL

Objective To determine the utility and cost-effectiveness of urine CMV PCR testing among infants with OFC $<9^{\text {th }}$ centile. To evaluate the frequency of alternative aetiologies of microcephaly in tested infants

Methods A retrospective review was conducted on all infants with a diagnosis of microcephaly for whom urine for CMV or TORCH screen was sent during July 2017-July 2018 in Coombe Women and Infants University Hospital ${ }^{1}$, Dublin, Ireland. Data was collected for various characteristics such as gestational age, ethnicity, parity, mother's serology, mother's lifestyle, reason of screening, anthropometric measurement/ Centile and cranial ultra sound result.

Results Between July 2017-July 2018, out of 8200 infants born in CWIUH, $72(0.87 \%)$ infants were screened. Of these, 56 infants had urine for CMV performed, and 16 infants had both TORCH titer and Urine for CMV testing performed. There were only 3 positive CMV cases (4.16\%). Male infants were $32(44.44 \%)$ and female infants were 40 (55.55\%). 25 infants were first born baby in their family. Mothers of 33 infants were drinking, smoking or using drugs during pregnancy. 29 infants underwent cranial ultrasound, of which 9 had abnormal results.

Conclusions By plotting on actual GA rather than on term line for term babies, we could avoid screening of $12.5 \%$ babies. Ethnicity factor is irrelevant. Considering the low incidence of congenital CMV infection (0.8\%), current local guidelines for congenital CMV infection screening is cost effective (yield $4.16 \%$ ). This study serves as a reminder to periodically examine testing practices and patient population to maximize cost-effectiveness.

\section{P442 ECHOCARDIOGRAPHY IN THE TERTIARY SURGICAL NEONATAL INTENSIVE CARE UNIT - A SERVICE EVALUATION PROJECT}

Katherine Millard, Emma Spencer*. Queen's Medical Centre, Nottingham, UK

\subsection{6/archdischild-2019-epa.778}

Introduction The use of echocardiography in neonatal care is currently the subject of significant and growing interest. It is well-recognised that there is wide variation in the approach to neonatal circulatory management, and that many of the commonly-assessed clinical parameters are of limited value in assessing adequacy of circulation. Much of the recent research interest has focussed on the potential uses of echocardiography in haemodynamic assessment of the preterm infant. However, the diverse group of newborn infants with surgical problems are also prone to cardiovascular compromise, particularly in the perioperative period. Additionally they may have structural cardiac defects, commonly as part of a syndrome or association. The echocardiographic needs of this cohort may therefore be different from those of the typical preterm population.

Aim The purpose of this study was to evaluate the use of echocardiography in a tertiary surgical neonatal intensive care unit to better understand current practice and the needs of the population, and to inform future service planning.

Methods We retrospectively reviewed all echocardiograms performed on our 17-cot unit over a 12-month period from November 2018 to October 2018. Demographic and clinical data were collected for all patients.

Results Over the study period 182 echocardiograms were performed on 122 infants. Echocardiograms were performed primarily by technicians, with $82 \%$ being performed within one day of the request being submitted. $43 \%$ of studies were carried out in term infants, with $52 \%$ taking place within the first three days of life. The most common indications were evaluation of a murmur in a preterm infant (22\%), known congenital or genetic anomalies (20\%) and functional cardiac assessment (13\%). A PDA was identified in 59\% of initial scans. Structural anomalies identified included ASD (27\%), VSD (17\%), AVSD (0.8\%), Tetralogy of Fallot (2.5\%) and coarctation of the aorta $(0.8 \%)$. Abnormal findings related to function or haemodynamics were reported in $19 \%$.

Conclusions Echocardiography is a commonly-performed investigation in a tertiary surgical neonatal intensive care unit. Both structural and functional assessments are important in the care of infants with surgical problems. This study provides baseline data on current practice and suggests a need for surgical neonatal services to consider adequate provision for echocardiography in any future service planning.

\section{P443 HEREDITARY THROMBOPHILIA IN NEWBORN}

Afef Ben Thabet, Rim Zaaghdoud, Manel Charfi, Chiraz Regaieg*, Amel Ben Hmed, Nedia Hmida, Amira Bouraoui, Abdellatif Gargouri. Department of neonatology, Hedi Chaker Hospital, Sfax, Tunisia

\subsection{6/archdischild-2019-epa.779}

Background Hereditary Thrombophilia refers to the propensity to develop thrombosis. A homozygous abnormality or combination of two heterozygous abnormal factors can lead to clinically apparent thrombotic disorders at an early age. The aim of our study was to analyse the epidemiology, clinical presentation, causes, and management of hereditary thrombophilia in newborn.

Methods It's a retrospective study of all cases of hereditary thrombophilia registered in the neonatal intensive care unit of Sfax between 2014 and 2017.

Results Seven full term newborns were included. They were 5 female and 2 male. Common clinical features were seizures, acute ischemic limb, mesenteric ischemia and skin necrosis 
(mean age of onset $=5.21$ days). Imaging identified stroke in 6 cases, subrenal aorta thrombosis in one case. We identified one case of protein $\mathrm{S}$ defesciency, 4 cases of isolated factor $\mathrm{V}$ leiden mutation, one case of isolated hyperhomocysteinemia and one case of combined factor V Leiden and hyperhomocysteinemia. The last one was presented with multiple cerebral and abdominal thrombosis. Family screening was performed in 3 cases. Treatment was based on Fresh frozen plasma transfusion in newborn who had severe protein C deficiency. None of our patients was treated with thrombolysis. During followup, there was no recurrence of thrombotic events. Three patients had neurological deficit. Two newborns died of disseminated intravascular coagulation.

Conclusions Thrombotic disorders at an early age should lead to performing thrombophilia testing. Family screening is essential to detect asymptomatic deficiency. Clinical features and treatment depend on thrombosis localization and extension.

\section{P444 MANAGEMENT OF ABDOMINAL MASSES IN THE NEWBORN: EXPERIENCE OF THE NEONATOLOGY DEPARTMENT OF SFAX (TUNISIA)}

${ }^{1}$ Chiraz Regaieg*, ${ }^{1}$ Rim Zaghdoud, ${ }^{2}$ Hayet Zitouni, ${ }^{1}$ Amel Ben Hmed, ${ }^{1}$ Manel Charfi, ${ }^{1}$ Amira Bouraoui, ${ }^{1}$ Nedia Hmida, ${ }^{2}$ Riadh Mhiri, ${ }^{1}$ Afef Ben Thabet, ${ }^{1}$ Abdellatif Gargouri. ${ }^{1}$ Department of neonatology, Hedi Chaker Hospital, Sfax, Tunisia; ${ }^{2}$ Department of pediatric surgery, Sfax, Tunisia

\subsection{6/archdischild-2019-epa.780}

Background Abdominal masses in neonates reflect a wide spectrum of diseases, from lesions that can cause significant morbidity and mortality, to conditions readily corrected surgically, to entities which may be safely observed.

Objective To evaluate epidemiology, clinical features, management and outcome of abdominal masses in the newborn.

Methods It's a retrospective study of all cases of abdominal masses registered in the neonatology department of Sfax between 2004 and 2019 .

Results Thirteen patients were included in the study. A female predominance was noted (sex ratio $=0.18$ ). Antenatal diagnosis was made in 10 cases. Seven patients were born via cesarean section. The mean gestational age was 37.7 weeks. Mean birth weight was $3160 \mathrm{~g}$. Three patients had fetal acute suffering and respiratory distress. The most frequent physical finding was palpable abdominal mass $(n=9)$. Ultrasonography ( $\mathrm{n}=13)$, abdominal scan $(\mathrm{n}=3)$ and MRI $(\mathrm{n}=4)$ were used for diagnosis. Tumor sizes ranged from 4.6 to $10 \mathrm{~cm}$. We had identified renal cystic lymphangioma $(n=1)$, Infantile myofibromatosis $(n=1)$, ileal duplication $(n=3)$, hydrocolpos $(n=4)$ and ovrian cysts $(n=4)$. Total resection was the treatment for ileal duplication ,ovarian cysts and lymphangioma cysts cases. The newborn with infantile myofibromatosis received medical treatment (vincristine) after incomplete resection. The treatment of hyrocolpos was based on simple hymenotomy in two cases and laparotomy in the other two complicated cases. Mean follow-up time was 24 months. Only one patient who had giant hydrocolpos died of refractory shock and acute kidney failure 3 days after surgery.

Conclusions Most neonatal abdominal masses are due to benign lesions. Some of them may provide diagnostic difficulties. Most of masses require surgical treatment, which can be safely performed in small infants by trained personnel. However genuine controversy exists in the management of some lesions including infantile myofibromatosis.

\section{P445 \\ PLANNNG FOR THE NEW CHILDREN'S HOSPITAL: ANALYSIS OF PATIENT POPULATION AND RESOURCE USE IN THE NEONATAL UNIT OF TEMPLE STREET CHILDREN'S UNIVERSITY HOSPITAL}

1,25inead Brannick* ${ }^{3}$ Grace Crilly, $1,4,5 \mathrm{Naomi}$ McCallion. 'Temple Street Children's University Hospital, Dublin, Ireland; ${ }^{2}$ National Maternity Hospital, Dublin, Ireland; ${ }^{3}$ School of Medicine and Medical Science, University College Dublin, Dublin, Ireland; ${ }^{4}$ The Rotunda Hospital, Dublin, Ireland; ${ }^{5}$ School of Medicine, Royal College of Surgeons Ireland, Dublin, Ireland

\subsection{6/archdischild-2019-epa.781}

Aims The neonatal service of Temple Street Children's University Hospital (TSCUH) includes St. Michael's B ward (SMB; 8 beds) and the HDU (6 beds). Admissions to the HDU are restricted to transfers from maternity hospitals (Brennan \& Murphy, 2018). The new children's hospital ( $\mathrm{NCH}$ ) will establish a single entity to integrate the services currently provided by TSCUH, OLCHC, and the NCH Tallaght (Department of Health, 2017). The aim of this study is to produce qualitative analysis of the patient population/resource use in the neonatal $\mathrm{HDU} / \mathrm{SMB}$ in order to plan for delivery of care in the $\mathrm{NCH}$. Methods Using the nursing admissions books, data was retrospectively collected for 6 months of admissions (July to December 2017).

Results In the HDU (N=59), 15\% of patients had a CGA of $<37 / 40$. The median age was 5 days; the median LOS was 8 days (range: 1 - 125 days). $71 \%$ of patients were jointly admitted by Neonatology/surgical specialty. The most common diagnoses were myelomeningocoele (14\%) and TOF (12\%). $19 \%$ of patients required NIV; $27 \%$ received TPN.

In SMB $(\mathrm{N}=279), 2 \%$ of patients had a CGA of $<37 / 40$. The median age was 6 weeks; the median LOS was 2 days (range: 1 - 22 days). $71 \%$ of patients were admitted under the care of General Paediatrics. The most common diagnosis was bronchiolitis $(22 \%)$ with a clear seasonal trend observed. $9 \%$ of patients required NIV.

Conclusion The neonatal ward in TSCUH offers a unique service, allowing access to specialist care that is not available in the maternity hospitals, and providing a high level of support to term/premature newborns. It is clear that a specialised neonatal ward is necessary to safely care for this patient population, along with separation from the significant burden of infection that is present on high-turnover general wards. We recommend the inclusion of a similar unit in the $\mathrm{NCH}$.

\section{REFERENCES}

1. Brennan, K, Murphy, J. (2018, March 9). Admissions criteria St Michaels B High Dependency Unit. Dublin: Temple Street Children's University Hospital.

2. Department of Health (2017). The new children's hospital. Retrieved June 2, 2018, from https://health.gov.ie/future-health/reforming-our-hospitals/the-newchildrens-hospital/

\section{P446 ASSOCIATION OF MATERNAL HEMOGLOBIN LEVEL WITH GESTATIONAL AGE AND BIRTH WEIGHT OF BABIES}

\footnotetext{
1,2Manoj Kumar Chaudhary*, ${ }^{1}$ Nisha keshari Bhatta, ${ }^{1}$ Mohan Chandra Regmi, ${ }^{1}$ Rupa Singh,

${ }^{1}$ Paricha Upadhyay. ${ }^{1}$ BPKIHS, Dharan, Nepal; ${ }^{2}$ PKMMH, Karjanha, Nepal
}

\subsection{6/archdischild-2019-epa.782}

Background Two million people, that is over 30\% of world's population is anemic. In Nepal prevalence of anemia in pregnancy is $42 \%$ and low birth weight varies from 12 to 15 